\title{
Prevalence of metacarpophalangeal sesamoid bones of the hand in Turkish population
}

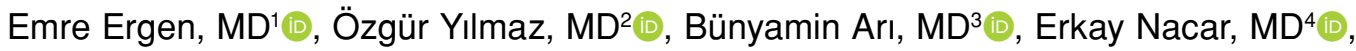 \\ Ayla Özaydoğdu Çimen, $\mathrm{MD}^{5}{ }^{\mathbb{D}}$, Kadir Ertem, MD ${ }^{1}$, Okan Aslantürk, $\mathrm{MD}^{6} \mathbb{B}$ \\ ${ }^{1}$ Department of Orthopedics and Traumatology, Inonü University Faculty of Medicine, Malatya, Turkey \\ ${ }^{2}$ Department of Orthopedics and Traumatology, Elazığ Fethi Sekin City Hospital, Elazı̆̆, Turkey \\ ${ }^{3}$ Department of Orthopedics and Traumatology, Turgut Özal University Faculty of Medicine, Malatya, Turkey \\ ${ }^{4}$ Karabük University, Public Health, Karabük, Turkey \\ ${ }^{5}$ Department of Radiology, Malatya Training and Research Hospital, Malatya, Turkey \\ ${ }^{6}$ Department of Orthopedics and Traumatology, Malatya Training and Research Hospital, Malatya, Turkey
}

The sesamoid term was first used by Galen. ${ }^{[1]}$ Sesamoid bones are described as small, oval or round-shaped bones located in the joint capsule or under tendons of the feet and hands. They can be also found in the wrist (pisiformis) and knee (patella, fabella and cyamella).

Functions of the sesamoid bones of the hand are still controversial. ${ }^{[2]}$ The main hypotheses proposed for functions of sesamoids are as follows: (i) reduction of tendon friction, (ii) protection of tendon, (iii) reduction of pressure, and (iv) maintaining the position of flexor pollicis longus. ${ }^{[2,3]}$

Plain radiographs, postmortem studies, ultrasound (US) and digital tomosynthesis (DTS) were used

Received: March 16, 2021

Accepted: April 272021

Published online: June 11, 2021

Correspondence: Okan Aslantürk, MD. Malatya Eğitim ve Araştırma Hastanesi Ortopedi ve Travmatoloji Kliniği, 44090 Yeşilyurt, Malatya, Türkiye.

E-mail: okaslanturk@hotmail.com

Doi: 10.52312/jdrs.2021.79

Citation: Ergen E, Yılmaz Ö, Arı B, Nacar E, Özaydoğdu Çimen A, Ertem $\mathrm{K}$, et al. Prevalence of metacarpophalangeal sesamoid bones of the hand in Turkish population. It Dis Relat Surg 2021;32(2):391-396.

(อ2021 All right reserved by the Turkish Joint Diseases Foundation

This is an open access article under the terms of the Creative Commons Attribution-NonCommercial License, which permits use, distribution and reproduction in any medium, provided the original work is properly cited and is not used for commercial purposes (http://creativecommons.org/licenses/by-nc/4.0/).

\section{ABSTRACT}

Objectives: This study aims to investigate the prevalence and location of the metacarpophalangeal (MCP) sesamoid bones using computed tomography (CT) images.

Patients and methods: A total of 767 hands of 735 patients (503 males, 232 females; mean age: 36.9 17.0 years; range, 18 to 105 years) obtained from picture archiving and communication system were retrospectively analyzed between January 2016 and December 2019. The sesamoid bones of MCP joints I, II, III, IV, and V were recorded. Data including age, sex, side, number, pathologies, and location of the sesamoid bones were recorded.

Results: The prevalence of sesamoid bones was found to be $100 \%, 37.61 \%, 1.17 \%, 0.5 \%$, and $80 \%$ in MCP I, II, III, IV, and V, respectively. There was no significant correlation between the sex of the patient and presence of sesamoid bone at MCP II or MCP $\mathrm{V}(\mathrm{p}>0.970$ and $\mathrm{p}=0.176$, respectively). The presence of sesamoid bone at MCP II was statistically significantly correlated with the presence of sesamoid bone at MCP V $(\mathrm{p}<0.001)$. There was no statistically significant difference in the side and sesamoid prevalence in the remaining 703 patients $(\mathrm{p}>0.05)$.

Conclusion: The prevalence of MCP V sesamoid bone is higher than previous studies from our country. The CT of hand can be used to determine sesamoid fractures and degenerative conditions of sesamoids.

Keywords: Computed tomography, hand, prevalence, sesamoid fracture, sesamoid bone.

in previous studies to investigate morphology and prevalence of hand sesamoid bones. ${ }^{[1,3-9]}$ Distinguishing the sesamoid bones can be difficult in the plain radiographs due to the overlapping bones. Ultrasound is user-dependent and not an ideal technique to evaluate bony structures. Digital 
tomosynthesis is not available in every center and do not produce high-quality images as computed tomography (CT). Cadaveric studies have limited number of subjects, but have certain advantages of morphological evaluation.

To the best of our knowledge, there is no study utilizing CT to investigate the hand sesamoid bones. In the present study, we aimed to investigate the prevalence and location of MCP sesamoid bone of the hand using CT.

\section{PATIENTS AND METHODS}

This single-center, retrospective study was conducted at Malatya Training and Research Hospital, Department of Orthopaedics and Traumatology between January 2016 and December 2019. The upper extremity CT scans obtained from picture archiving and communication system (PACS) were retrospectively reviewed. Inclusion criteria were as follows: age $>18$ years, hands in outstretched position, and $\mathrm{CT}$ scans containing proximal and distal of all MCP joints. Those younger than 18 years old, hands not in the neutral outstretched position due to CT topogram, and images not containing all MCP joints were excluded from the study. Finally, a total of 767 hands of 735 patients (503 males, 232 females; mean age: $36.9 \pm 17.0$ years; range, 18 to 105 years) were included. The study protocol was approved by the Inonü University Faculty of Medicine Ethics Committee (2020/1244). The study was conducted in accordance with the ethical principles of the Declaration of Helsinki.

All CT images were reviewed by an experienced orthopedic surgeon and radiologist. Data including age and sex of the patient, the side, number, and location of the sesamoid bone of the MCP joint, sesamoid and distal metacarpal morphology, and fractures of the sesamoid bones were recorded.

\section{Statistical analysis}

Statistical analysis was performed using the IBM SPSS version 20.0 software (IBM Corp., Armonk, NY, USA). Descriptive data were expressed in mean \pm standard deviation (SD), median (min-max)

\begin{tabular}{|c|c|c|c|}
\hline \multicolumn{4}{|c|}{$\begin{array}{c}\text { TABLE I } \\
\text { Patients data }\end{array}$} \\
\hline & $n$ & Median & Min-Max \\
\hline Number of subjects & 735 & & \\
\hline Number of hands & 767 & & \\
\hline Age (year) & & 36.9 & $18-105$ \\
\hline \multicolumn{4}{|l|}{ Sex } \\
\hline Male & 503 & & \\
\hline Female & 232 & & \\
\hline \multicolumn{4}{|l|}{ Side } \\
\hline Right & 303 & & \\
\hline Left & 400 & & \\
\hline Bilateral & 32 & & \\
\hline
\end{tabular}

or number and frequency, where applicable. The chi-square test was used to analyze the categorical data. A $p$ value of $<0.05$ was considered statistically significant.

\section{RESULTS}

Of a total of 767 hands included in the study, 303 were right hands, 400 were left hands, and 32 were bilateral hands (Table I).

Totally 130 hands (16.9\%) had a sesamoid bone only at MCP I, while the remaining hands (83.1\%) had a sesamoid bone(s) at least at two MCP joints. Only two patients had sesamoid bones at all MCP joints. The prevalence of $\mathrm{MCP}$ sesamoid bone is summarized in Table II.

There was no significant correlation between sex of the patient and presence of sesamoid bone at MCP II or MCP V ( $>0.970$ and $p=0.176$, respectively). The presence of MCP III sesamoid bone was higher in females $(2.1 \%)$ than males $(0.7 \%)$, although it did not reach statistical significance $(p=0.14)$. The presence of sesamoid bone at MCP II was statistically significantly correlated with the presence of sesamoid bone at MCP $\mathrm{V}(\mathrm{p}<0.001)$.

Thirty-two of the patients had bilateral hand $\mathrm{CT}$ and there was no significant difference in the

\begin{tabular}{|lccccc|}
\hline \multicolumn{7}{c|}{ TABLE II } & & \\
& Prevalence of MCP sesamoids \\
& MCP I & MCP II & MCP III & MCP IV & MCP V \\
\hline Number & 767 & 287 & 9 & 4 & 80 \\
Prevalence (\%) & 100 & 37.61 & 1.3 & 0.5 & 615 \\
\hline MCP: Metacarpophalangeal. & & & & & \\
\hline
\end{tabular}




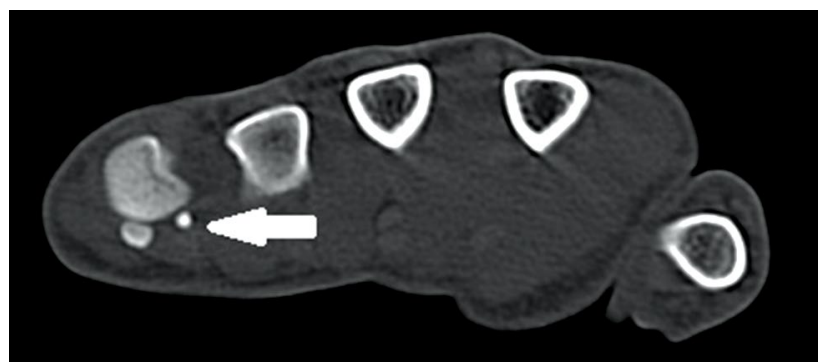

FIGURE 1. Rudimentary sesamoid bones of MCP V (white arrow).

MCP: Metacarpophalangeal.

number of sesamoid bones at MCP between each side. There was no statistically significant difference in the side and sesamoid prevalence in the remaining 703 patients $(\mathrm{p}>0.05)$. In 29 patients, the sesamoid bones were rudimentary (Figure 1).

\section{Features of sesamoid bones and distal metacarpus for MCP I}

The prevalence of at least one sesamoid bone at MCP I was $100 \%$ (767/767). Three patients had one sesamoid bone, two patients (one was bilateral) had bipartite sesamoid, and the remaining 761 hands of 730 patients had two sesamoid bones at MCP I (Figure 2).

In the patients with two sesamoid bones, one was located on ulnar side and the other on radial side. Sesamoid bone was located on radial side, when there was one sesamoid. Bipartite ones were also located on radial side (Figure 2). Ulnar sesamoid was round-shaped, while the radial one was oval-shaped and larger (Figure 2). However, when there was a bipartite sesamoid bone, the ulnar one was larger. The shape of the distal metacarpus was concave in all patients. There was no rudimentary sesamoid bone in MCP I.

\section{Features of sesamoid bones and distal metacarpus for MCP II}

The prevalence of sesamoid bone at MCP II was $37.61 \%$ (287/767). There was one sesamoid bone on the radial side in 284 hands (Figure 3). Two patients had two sesamoid bones at MCP II. Both patients had one on ulnar and one on radial side.
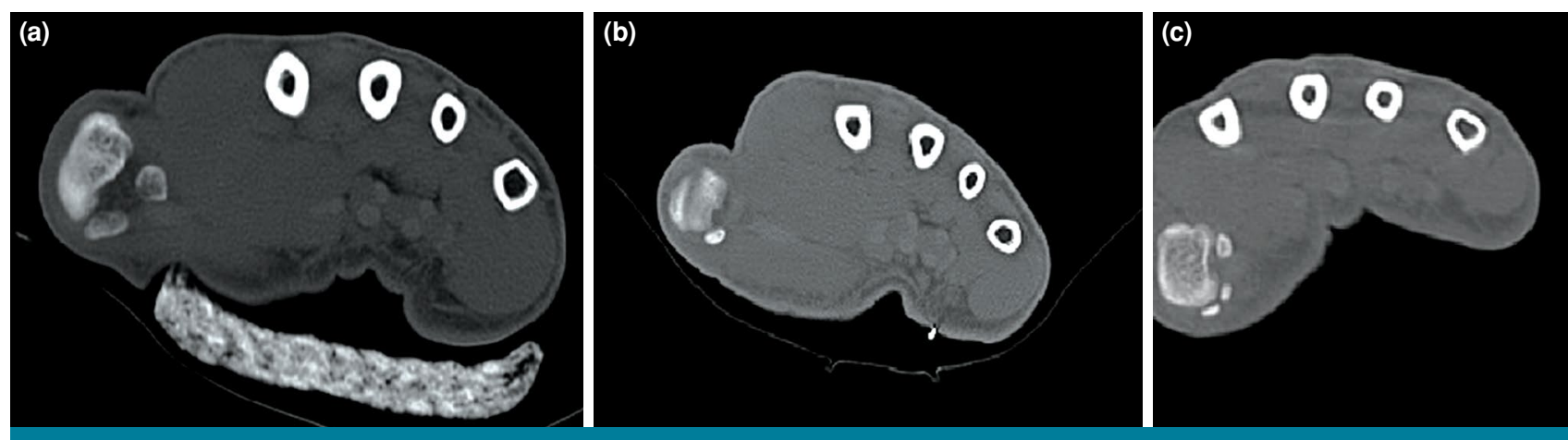

FIGURE 2. (a) An oval sesamoid bone on radial side and a round sesamoid bone on ulnar side. (b) Computed tomography images of patient with a single sesamoid bone on radial side of MCP I. (c) Bipartite radial sesamoid bone.

MCP: Metacarpophalangeal.
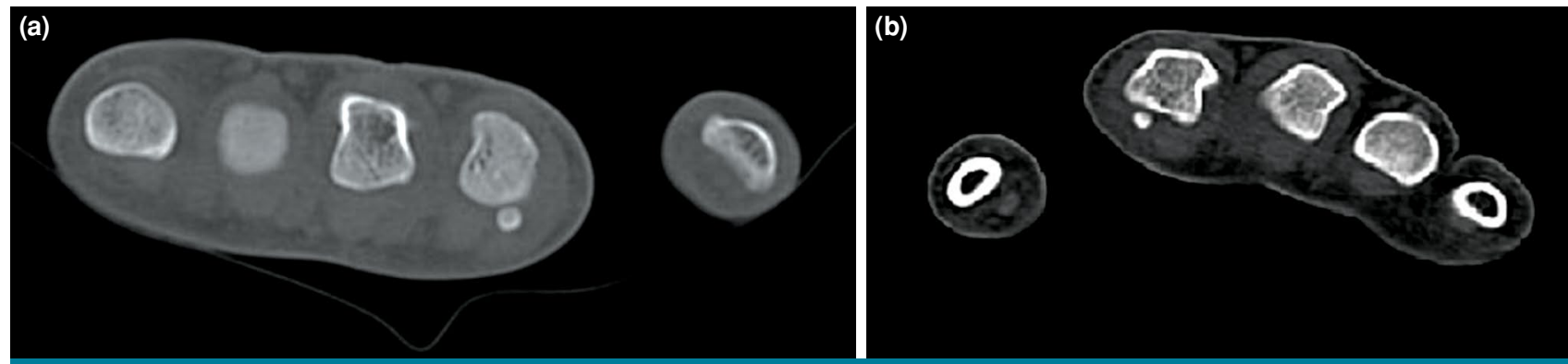

FIGURE 3. (a) A sesamoid bone of MCP II located on radial side, (b) centrally located sesamoid bone. Shape of distal metacarpus differed due to location of sesamoid bone.

MCP: Metacarpophalangeal. 

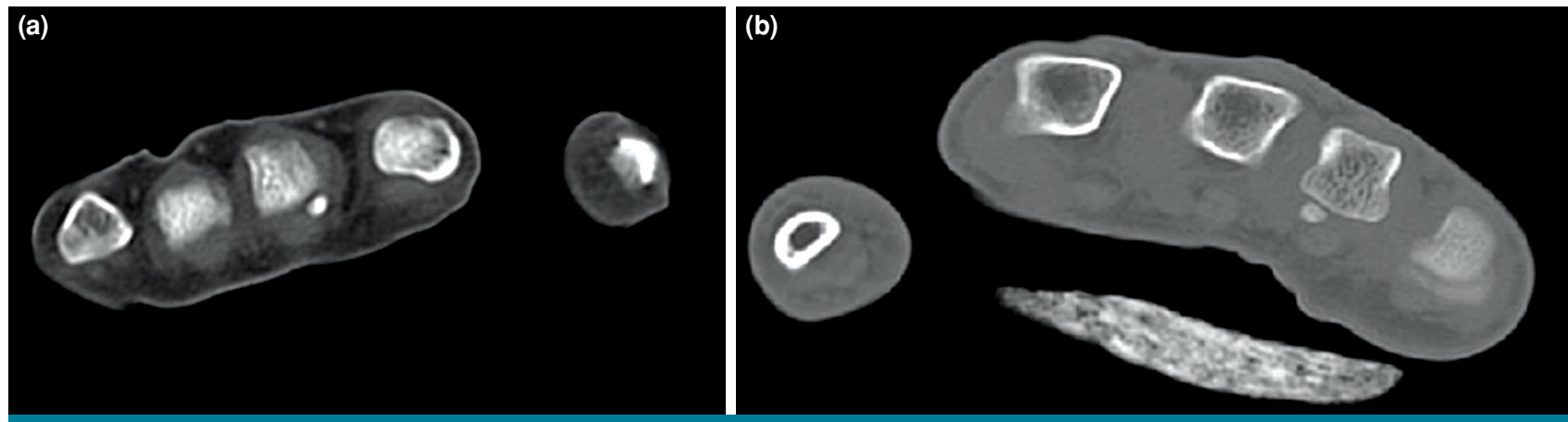

FIGURE 4. A sesamoid bone of (a) MCP III and (b) MCP IV. MCP: Metacarpophalangeal.

The shape of the sesamoid bone at MCP II was round. The shape of distal metacarpus was different due to location of sesamoid bone. Distal corner of the metacarpus was more convex, if the sesamoid bone was placed more laterally and it was flat or concave, if the sesamoid bone was located more centrally (Figure 3). Six of 286 sesamoid bones of MCP II were rudimentary.

\section{Features of sesamoid bones and distal metacarpus} for MCP III

The prevalence of sesamoid bone at MCP III was $1.3 \%$ (10/767). Five of patients (6 hands) were female and four males. Two of 10 bones were rudimentary. The shape of sesamoid bone was round. The sesamoid bone was located on radial side (Figure 4). The shape of the distal metacarpus was more convex, when the sesamoid bone was placed on the corner.

\section{Features of sesamoid bones and distal metacarpus for MCP IV}

The prevalence of sesamoid bone at MCP III was $0.5 \%$ (4/767). All patients were male. One patient had two sesamoid bones at MCP IV. One of four bones was rudimentary. Sesamoid bone was located on radial side and round-shaped (Figure 4). The shape of the distal metacarpus was more convex, when the sesamoid bone was placed on the corner.

\section{Features of sesamoid bones and distal metacarpus for MCP V}

The prevalence of sesamoid bone at MCP $\mathrm{V}$ was $80 \%$ (615/767). There were two sesamoid bones in MCP V in 24 (3.9\%) of 615 hands (Figure 5). Twenty of 615 sesamoid bones were rudimentary. In patients with two sesamoid bones, one was located on ulnar side and the other on radial side (Figure 5). In the patients with one sesamoid bone, the bone was located on ulnar side (Figure 5). The sesamoid bone was round-shaped. The shape of the distal metacarpus was more convex, when the sesamoid bone was placed on radial or ulnar corner.

\section{Fracture of sesamoid bones}

In our study, we detected only three $(0.4 \%)$ sesamoid bone fractures. Two of them were ulnar
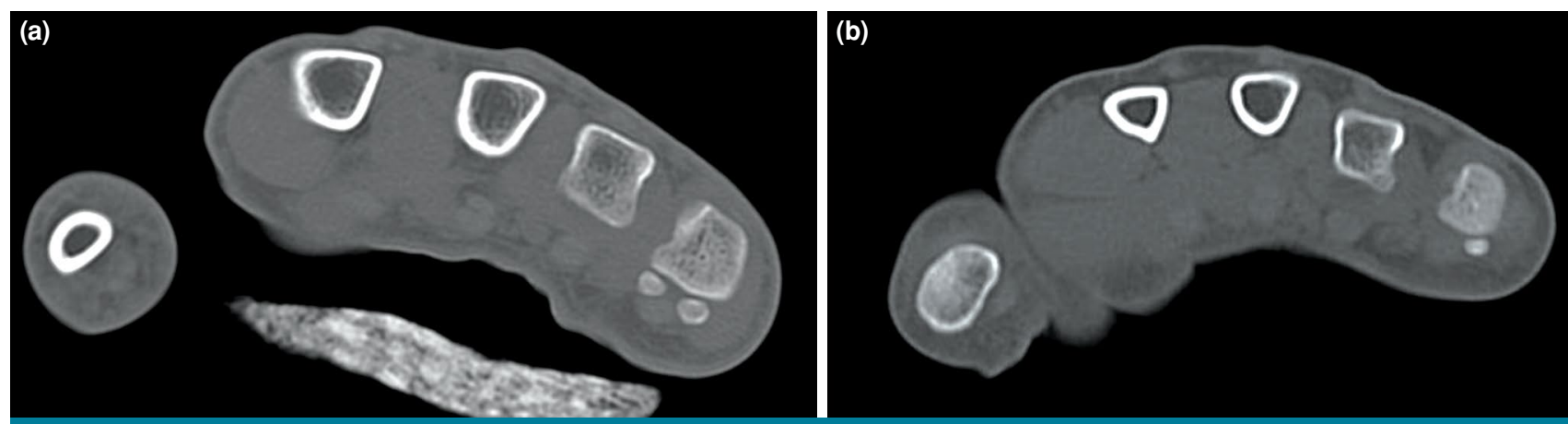

FIGURE 5. (a) A sesamoid bone of MCP V, when there were two sesamoid bones, one was located on ulnar and one on radial side. (b) When there was one sesamoid bone, the bone was located on ulnar side.

MCP: Metacarpophalangeal. 
sesamoid bone fractures of the thumb and one was radial sesamoid bone of the fifth digit.

\section{DISCUSSION}

In the literature, there are several studies regarding sesamoid bones of the hand. Previous studies assessing sesamoid bones usually used cadavers and radiological images (X-ray; US or DTS). The CT is more accurate for the assessment of bony structures. To the best of our knowledge, there is no previous study investigating sesamoid bones using CT. In current study, we assessed the prevalence, shape, and location of the sesamoid bones of MCP joints of the hand using $\mathrm{CT}$ images. The MCP V prevalence in our study was higher than previous studies from our country.

The presence of at least one sesamoid at the MCP I in different ethnicities has been reported by many authors. ${ }^{[3-9]}$ In a meta-analysis, Yammine ${ }^{[1]}$ reported a prevalence of sesamoid bone on radial, ulnar and both side of MCP I of $99.93 \%, 99.81 \%$, and $99.93 \%$, respectively. ${ }^{[1]}$ This slight difference may be caused by physiological agenesis of one sesamoid bone in some subjects, as three patients having one sesamoid bone in our study. To the best of our knowledge, there is only one thumb reported with absence of both sesamoid bones in the literature. ${ }^{[1]}$ In current study, the prevalence of sesamoid MCP I was $100 \%, 99.6 \%$, and $99.6 \%$ for radial, ulnar and both sides, respectively.

One of the previous cadaveric studies reported an ulnar sesamoid bone of the thumb which was larger than the radial one. ${ }^{[1]}$ However, the other studies reported larger radial sesamoid bones than ulnar sesamoid bones. ${ }^{[4]}$ In our study, we detected that radial sesamoid bones were larger than the ulnar ones, if not bipartite.

Bipartite sesamoid bone of the hand has been described only in a systematic review. ${ }^{[1]}$ Pfitzner (cited by Fawcett) reported three bipartite bones. ${ }^{[1]}$ However, we reviewed the Fawcett's article and there was no data about the bipartite sesamoid bone. Therefore, we believe that this is the first study reporting bipartite sesamoid of the hand. In our study, we detected three bipartite radial sesamoid bones of the thumb (one was bilateral) in two patients. However, we did not detect bipartite sesamoid bone at the other fingers.

The prevalence of the sesamoid bone at MCP II has been reported as an average of $48.4 \%$ (range, $4.8 \%$ to $64 \%)^{[1,8,10]}$ The sesamoid bone prevalence of MCP II has reported differently in different ethnicities. In Chinese, Caucasian, and Korean populations, the prevalence has been reported as $46 \%, 43.3 \%$, and $60.8 \%$, respectively. ${ }^{[3-5]}$ In two studies from Turkey, both reported a prevalence of sesamoid at MCP II as $36.6 \% .^{[6,7]}$ In the current study, we detected similar results as $37.61 \%$.

The reported prevalence of the sesamoid bone at MCP V in the literature ranges from 38.3 to $84.2 \%$ in different ethnicities. ${ }^{[1,3-5]}$ Two previous studies from Turkey reported a similar MCP V sesamoid prevalence of the hand as $53.2 \%$ and $62.5 \%$, respectively. ${ }^{[6,7]}$ The prevalence of MCP V sesamoid (80\%) in our study was significantly higher than previous two studies. This difference can be attributed to the accuracy of CT to determine the bony structures better. In their study, Koo et al. ${ }^{[4]}$ reported that plain radiography was not reliable to accurately determine the sesamoid bones. However, the main reason for the difference may be the region: both of previous studies were from the Western regions of Turkey, while our study is from the Eastern region of Turkey.

The prevalence of sesamoid bone at MCP III range from 0.8 to $5.3 \% .^{[4,5,8,9,11]}$ In previous studies from Turkey, Civan et al. ${ }^{[6]}$ and Kose et al. ${ }^{[7]}$ reported the prevalence as $1.1 \%$ and $1.3 \%$, respectively. We found a similar prevalence of $1.3 \%$ in our series. In their study, Kose et al. ${ }^{[7]}$ reported a higher prevalence of MCP III in female population. In contrast, Dharap et al. ${ }^{[12]}$ reported no sesamoid bone at MCP III in females. In our study, there was no significant difference in the MCP III sesamoid prevalence between the two sexes $(p=0.14)$, although females had a higher percentage of MCP III sesamoid bones than males ( $2.1 \%$ vs. $0.7 \%$, respectively).

The prevalence of MCP IV sesamoid has been reported as the lowest (range, 0 to 2.7\%) in most of previous series and reviews in the literature. ${ }^{[1,3-7]}$ In the current study, the MCP IV sesamoid prevalence was the lowest among all MCP with $0.5 \%$. All patients with a sesamoid bone at MCP IV were males. Only one previous study reported a sex distribution of MCP IV, and they found that only male participants had a sesamoid bone at MCP IV. ${ }^{[8]}$ Previous studies have shown that the sesamoid bone was located at ulnar side at MCP $\mathrm{IV}^{[3-7]}$ although it was located on radial side in our study.

In their study, Civan et al. ${ }^{[6]}$ reported that the presence of MCP II and V were statistically significantly higher in females. However, many other studies found no correlation between sex and presence of sesamoid bones. ${ }^{[1,9-12]}$ In the current study, we found no significant correlation between sex and 
the presence of sesamoid bone ( $p>0.970$ for MCP II and $p=0.176$ for MCP V). However, the presence of sesamoid bone at MCP II was statistically significantly correlated with the presence of sesamoid bone at MCP $\mathrm{V}(\mathrm{p}<0.001)$. To the best of our knowledge, this finding has not been reported previously.

The prevalence of the sesamoid fractures of the hand is still unclear. There is no study on this topic. Available data are mostly based on case reports. ${ }^{[13,14]}$ In the current study, we found three sesamoid bone fractures among 735 patients with a fracture prevalence of $0.4 \%$.

More intriguingly, we found that location of the sesamoid bone affected the shape of distal metacarpus in MCP II, III, IV, and V. When the sesamoid bone was located close the ulnar or radial corner of the distal metacarpus, the shape of distal metacarpus was convex. In contrast, when the sesamoid was located closer to the midline, the shape of distal metacarpus was concave. To the best of our knowledge, this finding has not been reported previously.

The retrospective design is the main limitation of our study. However, we believe that retrospective design did not have any influence on the sesamoid prevalence. Also, it would be ethical problem using having CT images of patients to determine only sesamoid bone prevalence due to high-dose radiation exposure. Therefore, we used already existing images from PACS and no imaging was performed to determine the sesamoid bone prevalence. The $\mathrm{CT}$ is a reference imaging tool to evaluate bony structures and, thus, we were able to determine even rudimentary sesamoid bones.

In conclusion, this is the first radiological study using CT images for hand sesamoid bones. According to our results, the prevalence of MCP V sesamoid bone is higher than previous studies from Turkey. The CT of hand can be used to determine sesamoid fractures and degenerative conditions of sesamoids.

\section{Declaration of conflicting interests}

The authors declared no conflicts of interest with respect to the authorship and/or publication of this article.

\section{Funding}

The authors received no financial support for the research and/or authorship of this article.

\section{REFERENCES}

1. Yammine K. The prevalence of the sesamoid bones of the hand: A systematic review and meta-analysis. Clin Anat 2014;27:1291-303.

2. Ozcanli H, Sekerci R, Keles N. Sesamoid disorders of the hand. J Hand Surg Am 2015;40:1231-2.

3. Dąbrowski K, Stankiewicz-Jóźwicka H, Kowalczyk A, Markuszewski M, Ciszek B. Ossa Sesamoidea - prevalence of sesamoid bones in human hands. Folia Morphol 2020;79:570-5.

4. Koo BS, Song Y, Sung YK, Lee S, Jun JB. Prevalence and distribution of sesamoid bones in the hand determined using digital tomosynthesis. Clin Anat 2017;30:608-13.

5. Chen W, Cheng J, Sun R, Zhang Z, Zhu Y, Ipaktchi K, et al. Prevalence and variation of sesamoid bones in the hand: A multi-center radiographic study. Int J Clin Exp Med 2015;8:11721-6.

6. Civan O, Şekerci R, Ercıktı N, Özer Ş, Güvenç İ, Keleş Çevik $\mathrm{N}$, et al. Sesamoid bones of the hand: A multicenter study. Jt Dis Relat Surg 2020;31:68-72.

7. Kose O, Guler F, Turan A, Canbora K, Akalin S. Prevalence and distribution of sesamoid bones of the hand. A radiographic study in Turkish subjects. Int. J. Morphol 2012;30:1094-9.

8. Ting LGY, San CEC, Ben N, Gerry HCH, Wai CC. Distribution of sesamoid bones in the hand-a study in the Chinese population. J. Orthop. Trauma Rehabilitation 2017;23:45-48.

9. Al Khabori H, Al Asmi M, Sirasanagandla SR, Al Rashdi F, Al Dhuhli H, Jaju S, et al. Prevalence and distribution of sesamoid bones of the hand in Omani subjects: A radiological study. Anat Sci Int 2021;96:79-86.

10. Msamati BC, Igbigbi PS. Radiographic appearance of sesamoid bones in the hands and feet of Malawian subjects. Clin Anat 2001;14:248-53.

11. Amar E, Rozenblat Y, Chechik O. Sesamoid and accessory bones of the hand--an epidemiologic survey in a Mediterranean population. Clin Anat 2011;24:183-7.

12. Dharap AS, Al-Hashimi H, Kassab S, Abu-Hijleh MF. Incidence and ossification of sesamoid bones in the hands and feet: A radiographic study in an Arab population. Clin Anat 2007;20:416-23.

13. Aslantürk $\mathrm{O}$, Ergen E. Fracture of the fifth finger sesamoid: A rare injury. Indian J Orthop 2019;53:616-7.

14. Connolly CM, Alsousou J, Hassan S, Williams NW. Don't avoid the sesamoids: Fracture to the ulnar sesamoid of the first metacarpophalangeal joint. Emerg Med J 2008;25:184. 